\title{
A Unified Framework for External Wrench Estimation, Interaction Control and Collision Reflexes for Flying Robots
}

\author{
Teodor Tomić ${ }^{1}$ and Sami Haddadin ${ }^{2}$
}

\begin{abstract}
Flying in unknown environments can lead to unwanted collisions with the environment. If not being accounted for, these may cause serious damage to the robot and/or its environment. Fast and robust collision detection combined with safe reaction is therefore essential in this context. Deliberate physical interaction may also be required in some applications. The robot can then switch into an interaction mode when contact occurs. The control loop must also be designed with interaction in mind. To implement these mechanisms, knowledge of environmental interaction forces is required. In principle, they may be measured or estimated. In this paper, we present a novel model-based method for external wrench estimation in flying robots. The estimation is based on proprioceptive sensors and the robot's dynamics model only. Using this estimate, we also design admittance and impedance controllers for sensitive and robust physical interaction. We also investigate the performance of our collision detection and reaction schemes in order to guarantee collision safety. Upon collision, we determine the collision location and normal located on the robot's geometric model. The method relies on the complete wrench information provided by our scheme. This allows applications such as tactile environment mapping.
\end{abstract}

\section{INTRODUCTION}

\section{A. Motivation and contribution}

Flying robots are being increasingly used in interaction and contact scenarios [1], [2], [3], [4], [5], such as tool force control and contact inspection. The applications typically require force sensing, which may be provided by a dedicated force sensor. However, this often limits the measurement to the contact point only. An external wrench estimate would make it possible to apply these methods without the need of additional sensors. A straightforward contact detection method can also be developed using the estimate. Using this context information, the robot can then switch into the appropriate mode. Flying robots are also increasingly applied in cluttered, unknown environments [6]. In this scenario, exteroceptive sensors can be used for mapping and collision avoidance. However, obstacle detection may sometimes fail or be inaccurate. For example, cameras and laser scanners typically see through windows and do not detect them as obstacles. Navigating in such environments therefore requires safe recovery from unforeseen collisions and contacts. Collision incidents could e.g. be stored in a map and used for replanning around the collided obstacle.

In this paper we present a unified framework for external wrench estimation, interaction control and collision detection

\footnotetext{
1 teodor.tomicedlr.de, German Aerospace Center (DLR) Robotics and Mechatronics Center (RMC), Münchner Straße 20, 82234 Weßling, Germany

2 sami.haddadineirt.uni-hannover.de, Institut für Regelungstechnik, Appelstr. 11, 30167 Hannover, Germany
}

for flying robots. First, we estimate the external force and torque acting on a flying robot based on the control input, a dynamics model and proprioceptive sensors. The estimator uses both the acceleration and angular velocity measurements. It is independent of the trajectory and the used controller, and makes no assumptions about the environment. Second, we design admittance and impedance control, which shape the robot's disturbance response to external forces. These methods can be used for physical interaction with the environment. Lastly, the wrench estimate enables us to perform the four essential phases of collision Fault Detection and Identification (FDI): detection, classification, isolation and reaction [7], [8]. We show that our proposed collision reactions greatly improve the robot's behavior upon collision. We outline how to use the external wrench estimate to obtain the collision location and its respective normal. This may e.g. be used as tactile mapping information.

\section{B. Related work}

External wrench estimation for flying robots has been investigated previously in the context of hybrid pose/wrench control [1]. Bellens et al. measured offline the forces and torques generated by the UAV while fixed to a base, under the whole range of control inputs. The estimated wrench is then functionally related to the control inputs and is only valid while the robot is in contact.

Physical interaction of flying robots with the environment has been addressed by several researchers. Albers et al. [9] have used a dedicated propeller for horizontal force control. However, they only used a feedforward signal to exert a contact force. Force control at a tooltip rigidly attached to a flying robot has more recently been investigated in [2], [3], [4]. Therein, the force is assumed to be measured by a force sensor. Further, Forte et al. [10] and Fumagalli et al. [4], [5] have applied impedance control to UAVs for contact inspection. They rely on the passivity properties of impedance control, and do not require an external force measurement.

Naldi et al. [11] have implemented a control loop supervisor for contact detection. Contact is assumed if the control error of the path following controller is above a threshold. The path is interpolated backwards until there is no collision anymore. No wrench estimation is performed. This makes the method trajectory and controller dependent. Briod et al. [12] use onboard accelerometers and small force sensors attached to elastic springs to detect collisions with the environment. The acceleration-based approach detects collisions when the acceleration magnitude is above a threshold. This is chosen 
higher than accelerations occuring during normal flight. The sensitivity of the method therefore depends significantly on the controller and the trajectory. When a collision occurs, the robot turns off the motors and relies on its robust mechanical design to land safely. It resumes flight opposite of the detected collision direction, which is used for tactile exploration.

This paper is organized as follows. In Section II we present the model and position control scheme of a quadrotor vehicle. Section III presents methods for external wrench estimation based on the UAV model. Section IV presents the admittance and impedance control methods that use the external wrench estimate. Collision detection and reaction strategies are investigated in Section V. Finally, in Section VI we present experimental results showing the effectiveness of the presented methods.

\section{Preliminaries: Modeling, POSition And ATTITUDE CONTROL}

\section{A. Modeling}

The equations of motion of a vertical takeoff and landing (VTOL) UAV about its center of mass can be written as

$$
\begin{aligned}
m \ddot{\boldsymbol{r}} & =m g \boldsymbol{e}_{3}+\boldsymbol{R}_{b i}^{T} \boldsymbol{f}+\boldsymbol{f}_{e} \\
\boldsymbol{I} \dot{\boldsymbol{\omega}} & =(\boldsymbol{I} \boldsymbol{\omega}) \times \boldsymbol{\omega}+\boldsymbol{m}+\boldsymbol{R}_{b i} \boldsymbol{m}_{e} \\
\dot{\boldsymbol{R}}_{b i} & =\boldsymbol{R}_{b i}(\boldsymbol{\omega}) \times
\end{aligned}
$$

where $m$ is the UAV mass, $\boldsymbol{r}=[x y z]^{T}$ is its position in the fixed North-East-Down (NED) inertial frame, $\boldsymbol{R}_{b i}$ is the rotation matrix from the inertial to the body frame, $\boldsymbol{I}$ is the moment of inertia, $g$ is the acceleration of gravity, $\omega$ is the UAV angular velocity, $\boldsymbol{e}_{3}$ is the $z$-axis unit vector, $f=\left[f_{x} f_{y} f_{z}\right]^{T}$ is the control force in the body frame, $\boldsymbol{f}_{e}=\left[f_{e, x} f_{e, y} f_{e, z}\right]^{T}$ is the external force in the inertial frame, $\boldsymbol{m}=\left[m_{x} m_{y} m_{z}\right]^{T}$ is the body-frame control torque, and $\boldsymbol{m}_{e}=\left[m_{e, x} m_{e, y} m_{e, z}\right]^{T}$ is the external torque in the inertial frame. The external wrench acting on the UAV in the inertial frame is $\boldsymbol{\tau}_{e}=\left[\boldsymbol{f}_{e} \boldsymbol{m}_{e}\right]^{T}$. The control wrench generated by the propellers in the body frame is $\boldsymbol{\tau}=[\boldsymbol{f} \boldsymbol{m}]^{T}$. The propeller angular velocities are obtained from $\tau=$ $\boldsymbol{B} \boldsymbol{u}$, where $\boldsymbol{B} \in \mathbb{R}^{6 \times N}$ is the control allocation matrix dependent on the number of propellers $N$, their locations on the UAV frame and their aerodynamic properties. The vector $\boldsymbol{u}$ contains squares of the required propeller angular velocities $\Omega$, with $u_{i}=\Omega_{i}^{2}$, and can be obtained e.g. by (pseudo-)inverting the matrix $\boldsymbol{B}$. In this paper we assume that the control input is exactly the wrench generated by the propellers. By writing the twist as $\boldsymbol{\nu}=[\dot{\boldsymbol{r}} \boldsymbol{\omega}]^{T}$, the equations of motion can be rewritten in Lagrange form as

$$
\boldsymbol{M} \dot{\boldsymbol{\nu}}+\boldsymbol{C}(\boldsymbol{\nu}) \boldsymbol{\nu}+\boldsymbol{D}(\boldsymbol{\nu}) \boldsymbol{\nu}+\boldsymbol{g}=\boldsymbol{J}^{T} \boldsymbol{\tau}+\boldsymbol{\tau}_{e},
$$

where $\boldsymbol{g}=\left[-m g \boldsymbol{e}_{3} \mathbf{0}\right]^{T}, \boldsymbol{J}=\operatorname{blockdiag}\left\{\boldsymbol{R}_{b i}, \boldsymbol{I}_{3 \times 3}\right\}$, and

$$
\boldsymbol{M}=\left[\begin{array}{cc}
m \boldsymbol{I}_{3 \times 3} & \mathbf{0}_{3 \times 3} \\
\mathbf{0}_{3 \times 3} & \boldsymbol{I}
\end{array}\right] \quad \boldsymbol{C}(\boldsymbol{\nu})=\left[\begin{array}{cc}
\mathbf{0}_{3 \times 3} & \mathbf{0}_{3 \times 3} \\
\mathbf{0}_{3 \times 3} & -(\boldsymbol{I} \boldsymbol{\omega}) \times
\end{array}\right] .
$$

Without loss of generality, the term $\boldsymbol{D}(\boldsymbol{\nu}) \boldsymbol{\nu}$ represents the aerodynamic drag forces.

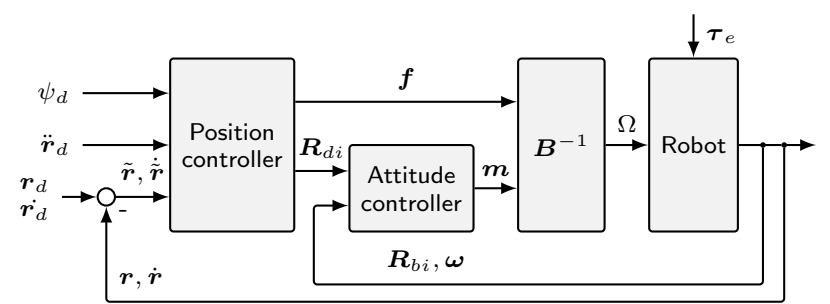

Fig. 1: Cascaded control structure for a flying robot. The inner attitude controller tracks the reference orientation. The position controller tracks the position and velocity by commanding the thrust vector. The yaw angle is free to command and comes directly from the reference trajectory.

\section{B. Position and Attitude Control}

For our quadrotor vehicle we employ a cascaded control structure [13], with an inner attitude controller, and an outer position tracking controller, as depicted in Fig. 1. The position controller tracks a desired position $\boldsymbol{r}_{d}=\left[\begin{array}{lll}x_{d} & y_{d} & z_{d}\end{array}\right]^{T}$, velocity $\dot{\boldsymbol{r}}_{d}$ and acceleration $\ddot{\boldsymbol{r}}_{d}$. The inertial control force is given by

$$
\boldsymbol{f}_{i}=\boldsymbol{R}_{d i}^{T} \boldsymbol{f}=m\left(\ddot{\boldsymbol{r}}_{d}-\boldsymbol{K}_{d} \dot{\tilde{\boldsymbol{r}}}-\boldsymbol{K}_{p} \tilde{\boldsymbol{r}}\right)-m g \boldsymbol{e}_{3},
$$

where $\boldsymbol{K}_{d} \in \mathbb{R}^{3 \times 3}$ is the diagonal positive definite damping gain, $\boldsymbol{K}_{p} \in \mathbb{R}^{3 \times 3}$ is the diagonal positive definite proportional gain, and $\tilde{\boldsymbol{r}}=\boldsymbol{r}-\boldsymbol{r}_{d}$ is the position error. The resulting control force in the body frame is $\boldsymbol{f}=\left[\begin{array}{ll}0 & 0-T\end{array}\right]^{T}$, where $T=\left\|\boldsymbol{f}_{i}\right\|$ is the total thrust produced by the propellers. Since the quadrotor can apply a force only in the body $z$-frame, it must be rotated so that the thrust vector coincides with $f_{i}$. The roll and pitch angles and the thrust are determined by the position controller. Because the quadrotor has four control inputs, the yaw angle $\psi$ is left free to be controlled. The required attitude $\boldsymbol{R}_{d i}$ is thus obtained from the force $\boldsymbol{f}_{i}$ and desired yaw angle $\psi_{d}$. The attitude is controlled by the control law

$$
\boldsymbol{m}=\boldsymbol{I}\left(-\boldsymbol{K}_{\omega} \boldsymbol{\omega}-c \boldsymbol{e}\right)-(\boldsymbol{I} \boldsymbol{\omega}) \times \boldsymbol{\omega},
$$

where $\boldsymbol{K}_{\omega} \in \mathbb{R}^{3 \times 3}$ is the diagonal positive definite damping gain, $c>0$ is the positive proportional gain, and $\boldsymbol{e}$ is the geometric attitude error vector obtained from $\tilde{\boldsymbol{R}}=\boldsymbol{R}_{d i}^{T} \boldsymbol{R}_{b i}$.

\section{EXTERNAL WRENCH ESTIMATION}

Knowledge of the system model and control laws can be used to estimate the external wrench acting on the robot [14]. We investigate two estimation methods - the momentumbased method which uses velocity information, and the acceleration-based method.

\section{A. Momentum-based estimation}

The generalized momentum $\boldsymbol{p}=\boldsymbol{M \nu}$ according to (4) satisfies

$$
\dot{\boldsymbol{p}}=\boldsymbol{M} \dot{\boldsymbol{\nu}}=\boldsymbol{J}^{T} \boldsymbol{\tau}+\boldsymbol{\tau}_{e}-\boldsymbol{N},
$$

where $\boldsymbol{N}=\boldsymbol{C}(\boldsymbol{\nu}) \boldsymbol{\nu}+\boldsymbol{D}(\boldsymbol{\nu}) \boldsymbol{\nu}+\boldsymbol{g}$. Following [14], we define a residual vector

$$
\boldsymbol{\rho}=\boldsymbol{K}_{I}\left[\boldsymbol{p}-\int\left(\boldsymbol{J}^{T} \boldsymbol{\tau}+\boldsymbol{\tau}_{e}-\boldsymbol{N}+\boldsymbol{\rho}\right) \mathrm{d} s\right]
$$


TABLE I: Measurements provided by sensors that are applicable to the presented wrench estimation methods. We are interested in the translational velocity $\dot{\boldsymbol{r}}$, acceleration $\ddot{\boldsymbol{r}}$, and angular velocity $\boldsymbol{\omega}$ and acceleration $\dot{\boldsymbol{\omega}}$. These can be directly measured $\bullet$, obtained numerically $\circ$, or estimated $\triangle$.

\begin{tabular}{|l||c|c|c|c|}
\hline Sensor & $\dot{\boldsymbol{r}}$ & $\boldsymbol{\omega}$ & $\ddot{\boldsymbol{r}}$ & $\dot{\boldsymbol{\omega}}$ \\
\hline \hline Accelerometer & - & - & $\bullet$ & - \\
Gyroscope & - & $\bullet$ & - & $\circ$ \\
PX4FLOW [15] & $\triangle$ & $\bullet$ & - & $\circ$ \\
IMU-odometry fusion [6] & $\triangle$ & $\bullet$ & $\bullet$ & $\circ$ \\
\hline
\end{tabular}

with positive diagonal $\boldsymbol{K}_{I} \in \mathbb{R}^{6 \times 6}$. By differentiating (8), we obtain the residual dynamics

$$
\dot{\boldsymbol{\rho}}=\boldsymbol{K}_{I} \boldsymbol{\tau}_{e}-\boldsymbol{K}_{I} \boldsymbol{\rho},
$$

which is a linear exponentially stable system driven by the external wrench $\tau_{e}$. Hence, $\rho$ will be the lowpass filtered reconstruction of $\tau_{e}$. Therefore, the estimated external wrench will be $\hat{\tau}_{e}=\boldsymbol{\rho}$. Note that this method requires the measurement or estimation of the twist $\nu$.

\section{B. Acceleration-based estimation}

The external wrench can be obtained directly from acceleration information. By rearranging terms in (4) we can algebraically calculate the external wrench as

$$
\hat{\boldsymbol{\tau}}_{e}=\boldsymbol{M} \dot{\boldsymbol{\nu}}+\boldsymbol{C}(\boldsymbol{\nu}) \boldsymbol{\nu}+\boldsymbol{D}(\boldsymbol{\nu}) \boldsymbol{\nu}+\boldsymbol{g}-\boldsymbol{\tau},
$$

where the acceleration $\dot{\nu}$ should be filtered due to sensor noise. This method is suitable for flying robots because the translational accelerations are directly measured by an onboard IMU.

\section{Hybrid estimation}

Table I shows measurement possibilities of the sensor values required for the presented wrench estimation methods. Obtaining a drift-free translational velocity requires exteroceptive sensors and a fusion algorithm. This greatly limits the applicability of the momentum based method for force estimation. Similarly, the angular acceleration can only be obtained through numerical differentiation. This reduces the quality of the torque estimate by the acceleration based method. By considering the directly measured values, it makes sense to combine the two presented methods. We use the acceleration method (10) for external force estimation, and the momentum method (7) for external torque estimation. The resulting estimator structure is depicted in Fig. 2. We lowpass filter the acceleration to reduce measurement noise. By combining both methods we can estimate the external wrench using proprioceptive sensors only. The hybrid estimator has the form

$$
\begin{aligned}
\hat{\boldsymbol{f}}_{e} & =m \boldsymbol{R}_{b i}^{T} \boldsymbol{a}^{b}-\boldsymbol{R}_{b i}^{T} \boldsymbol{f} \\
\hat{\boldsymbol{m}}_{e}^{b} & =\boldsymbol{K}_{I}^{m}\left(\boldsymbol{I} \boldsymbol{\omega}-\int_{0}^{t}\left(\boldsymbol{m}+(\boldsymbol{I} \boldsymbol{\omega}) \times \boldsymbol{\omega}-\hat{\boldsymbol{m}}_{e}^{b}\right) \mathrm{d} s\right) \\
\hat{\boldsymbol{m}}_{e} & =\boldsymbol{R}_{b i}^{T} \hat{\boldsymbol{m}}_{e}^{b}
\end{aligned}
$$

where $\boldsymbol{a}^{b}=\boldsymbol{R}_{b i}\left(\ddot{\boldsymbol{r}}-g \boldsymbol{e}_{3}\right)$ is the acceleration measured by an accelerometer in the center of mass expressed in the

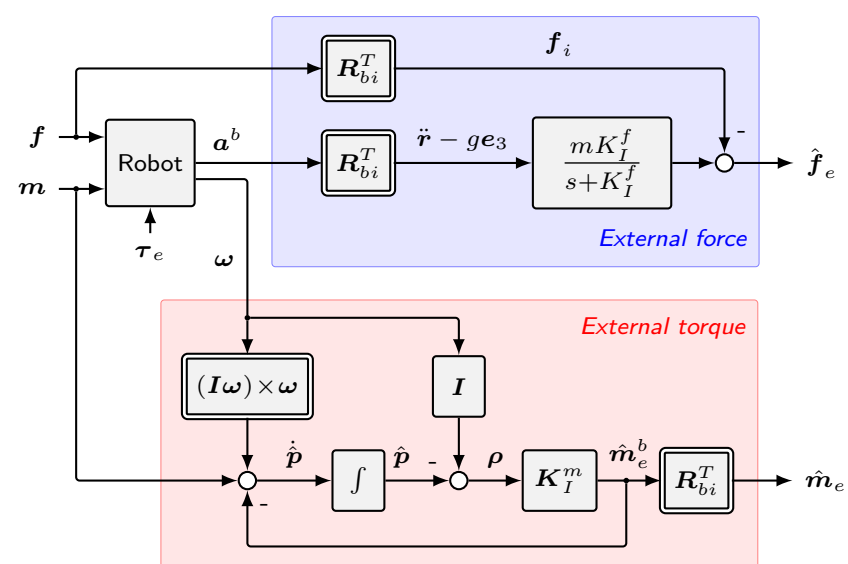

Fig. 2: Structure of the hybrid external wrench estimator. It uses the body acceleration $\ddot{\boldsymbol{r}}^{b}$ and angular velocity $\boldsymbol{\omega}$, both of which are measured directly by the onboard IMU. The estimated external wrench $\hat{\boldsymbol{\tau}}_{e}=\left[\begin{array}{ll}\hat{\boldsymbol{f}}_{e} & \hat{\boldsymbol{m}}_{e}\end{array}\right]^{T}$ is expressed in the inertial frame. The estimator gain $\boldsymbol{K}_{I}$ is split into its diagonal blocks $\boldsymbol{K}_{I}^{f}$ for the force and $\boldsymbol{K}_{I}^{m}$ for the torque components.

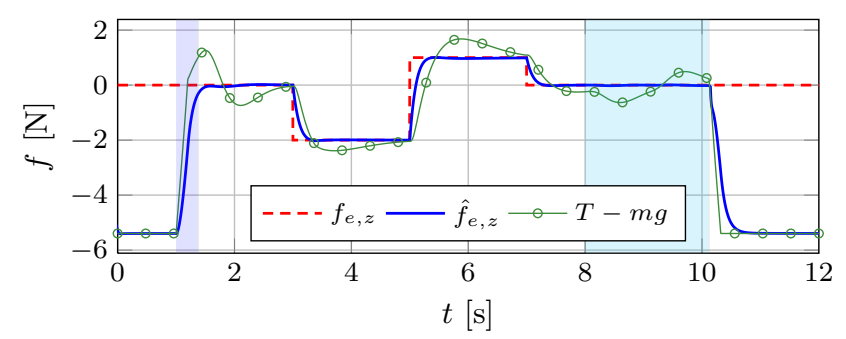

Fig. 3: External force estimation in the $\boldsymbol{e}_{3}$ (inertial $z$ ) direction with $m=0.55 \mathrm{~kg}$ and $K_{I}^{f}=10$. The shaded areas indicate takeoff and landing phases, respectively. The offset thrust input shows that the control input is taken into account. The estimated force is a lowpass filtered signal of the external force, as can be seen from the step response in flight. During contact with the ground, the ground force is estimated, with magnitude equal to the robot weight.

body frame, and $\hat{\boldsymbol{m}}_{e}^{b}$ is the estimated external torque, also expressed in the body frame. Note that an accelerometer also measures the acceleration of gravity. The estimator dynamics are linear and decoupled in both methods. Fig. 3 shows the response of the estimator to changes in the external force in the inertial $z$-direction.

\section{Discussion}

In general, the external wrench consists of the modeling error $\boldsymbol{\tau}_{m}$, disturbance wrench $\boldsymbol{\tau}_{d}$ and collision wrench $\boldsymbol{\tau}_{c}$, such that $\boldsymbol{\tau}_{e}=\boldsymbol{\tau}_{m}+\boldsymbol{\tau}_{d}+\boldsymbol{\tau}_{c}$. The wrench $\boldsymbol{\tau}_{c}$ may be measured by a force/torque sensor. However, in general it will not be possible to distinguish between the external wrench components. In this paper we will assume $\tau_{e}=\tau_{c}$. Hence, we assume that there are no modeling errors $\left(\boldsymbol{\tau}_{m}=\mathbf{0}\right)$ and no external disturbance is present $\left(\boldsymbol{\tau}_{d}=\mathbf{0}\right)$. Furthermore, the applied control wrench $\tau$ is required for external wrench estimation. This can not be directly measured in a flying robot during flight. We therefore assume that the commanded wrench is applied ideally and use the commanded wrench in the estimator. 


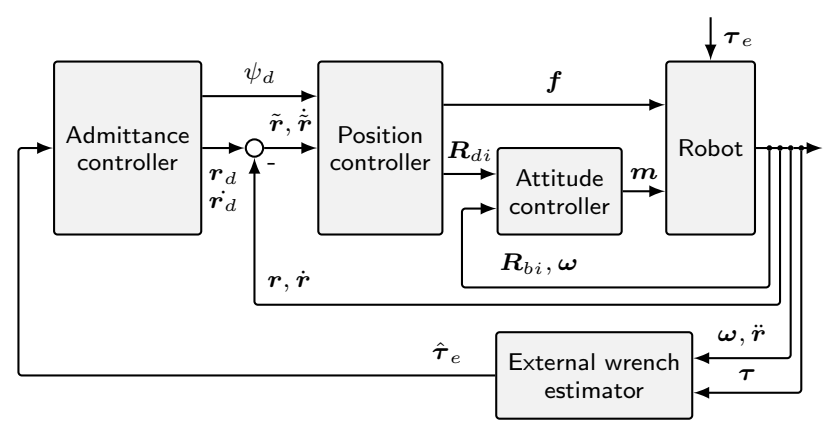

(a) Admittance controller (15) structure.

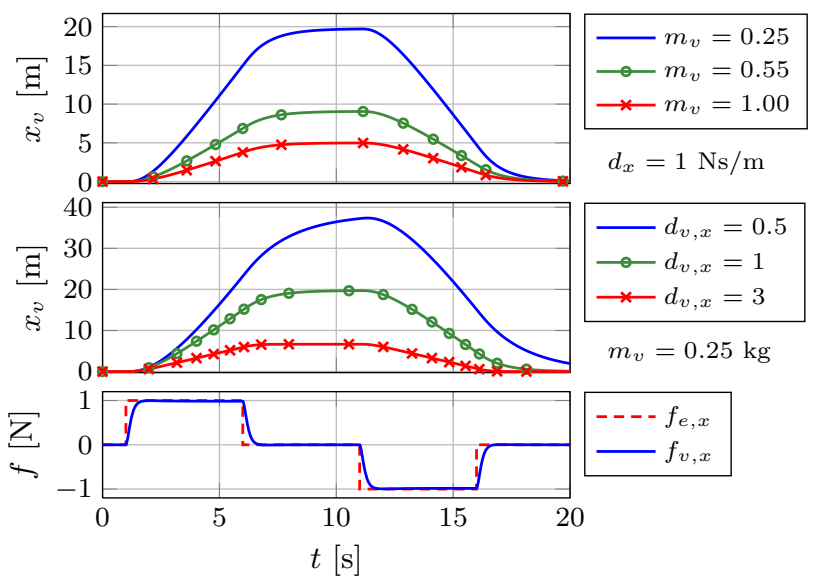

(b) Response of the admittance filter (14) in the inertial $x$-direction for different virtual inertia and damping parameters. The parameters determine the steady-state velocity of the virtual system. The resulting trajectory is tracked by the position controller.

Fig. 4: Structure and response of the admittance controller.

\section{INTERACTION CONTROL}

Now that we have an estimate of the external wrench, we can integrate this information into the robot control. Exemplarily, we show admittance control and impedance control approaches to obtain suitable interaction behavior using contact wrench information.

\section{A. Admittance control}

Admittance control basically generates a velocity command as a function of the contact wrench. The resulting trajectory may then be tracked using (5) and (6). In effect, this adds an additional cascade around the position control loop. The reference velocity and position may be generated using the virtual dynamical system

$$
\left[\begin{array}{cc}
m_{v} & 0 \\
\mathbf{0}_{3 \times 3} & I_{v, z}
\end{array}\right]\left[\begin{array}{c}
\ddot{\boldsymbol{r}}_{d} \\
\ddot{\psi}_{d}
\end{array}\right]+\boldsymbol{D}\left[\begin{array}{c}
\dot{\boldsymbol{r}}_{d} \\
\dot{\psi}_{d}
\end{array}\right]+\boldsymbol{K}\left[\begin{array}{c}
\boldsymbol{r}_{d}-\boldsymbol{r}_{d}^{*} \\
\psi_{d}-\psi_{d}^{*}
\end{array}\right]=\left[\begin{array}{c}
\hat{\boldsymbol{f}}_{e} \\
\hat{m}_{e, z}
\end{array}\right]
$$

where $m_{v}>0$ and $I_{v, z}>0$ are the virtual mass and inertia, $\boldsymbol{D}_{v} \in \mathbb{R}^{4 \times 4}$ is the positive definite diagonal virtual damping gain, $\boldsymbol{K}_{v} \in \mathbb{R}^{4 \times 4}$ is the positive diagonal virtual spring gain, $\boldsymbol{r}_{d}^{*}$ and $\psi_{d}^{*}$ are the optional desired setpoints, $\hat{\boldsymbol{f}}_{e}$ is the estimated external force, and $\hat{m}_{e, z}$ is the estimated external torque about the $z$-axis. The torque information is

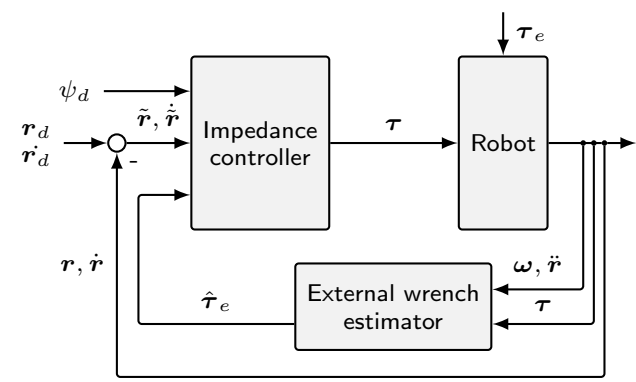

(a) Impedance controller (17) structure.

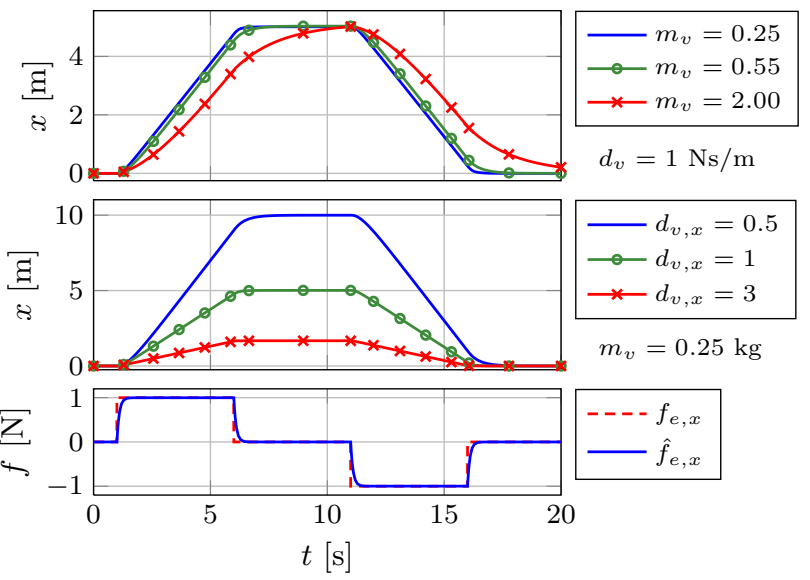

(b) Simulated response of the position impedance controller to an external force in the inertial $x$-direction, for varying virtual inertia and damping parameters. The real system mass is $m=0.55 \mathrm{~kg}$. The system is in damping mode, i.e. the vitual stiffness is set to $\boldsymbol{K}_{v}=\mathbf{0}$.

Fig. 5: Structure and response of the impedance controller.

used to implement an admittance on the yaw angle. The desired roll and pitch angles are commanded by the position controller, see Section II. They are therefore omitted from the admittance controller. Equation (14) can more concisely be written as

$$
\boldsymbol{M}_{v} \ddot{\boldsymbol{x}}_{v}+\boldsymbol{D}_{v} \dot{\boldsymbol{x}}_{v}+\boldsymbol{K}_{v} \tilde{\boldsymbol{x}}_{v}=\boldsymbol{\tau}_{v} .
$$

If we choose $\boldsymbol{K}_{v}=\mathbf{0}_{4 \times 4}$, a human operator can move the robot freely in space. The steady state velocity will then be

$$
\dot{\boldsymbol{x}}_{v, s s}=\boldsymbol{D}_{v} \boldsymbol{M}_{v}^{-1} \boldsymbol{\tau}_{v}
$$

which in turn can be used to design the desired behavior of the admittance system. The admittance control structure is shown in Fig. 4(a), and the response of (15) is depicted in Fig. 4(b). The admittance controller adds another cascade into the control loop. This makes it simple to implement the method on a system where a position controller is already available. However, the degree of the overall system is increased due to the wrench estimator dynamics and the virtual system (14). This complicates stability properties of the overall system, and adds significant delay dynamic, reducing the responsivness of the system significantly.

\section{B. Impedance control and inertia shaping}

For interaction control, a robot should ideally represent an impedance, as was argued in the seminal work of Hogan 
[16]. We define the target closed loop dynamics of (4) to be

$$
\boldsymbol{M}_{v} \dot{\boldsymbol{\nu}}+\boldsymbol{D}_{v} \tilde{\boldsymbol{\nu}}+\boldsymbol{K}_{v} \tilde{\boldsymbol{x}}=\boldsymbol{\tau}_{e} .
$$

where $\boldsymbol{M}_{v} \in \mathbb{R}^{6 \times 6}$ is the desired positive definite apparent inertia matrix, $\boldsymbol{D}_{v} \in \mathbb{R}^{6 \times 6}$ is the desired positive definite damping matrix, and $\boldsymbol{K}_{v} \in \mathbb{R}^{6 \times 6}$ is the desired positive definite stiffness matrix. The state and velocity errors are $\tilde{\boldsymbol{x}}=[\tilde{\boldsymbol{r}} \boldsymbol{e}]^{T}$ and $\tilde{\boldsymbol{\nu}}=\boldsymbol{\nu}-\boldsymbol{\nu}_{d}$, respectively. By inserting (16) into (4), the required control input is

$$
\boldsymbol{J}^{T} \boldsymbol{\tau}=\left(\boldsymbol{M} \boldsymbol{M}_{v}^{-1}-\boldsymbol{I}\right) \hat{\boldsymbol{\tau}}_{e}-\boldsymbol{M} \boldsymbol{M}_{v}^{-1}\left(\boldsymbol{D}_{v} \tilde{\boldsymbol{\nu}}+\boldsymbol{K}_{v} \tilde{\boldsymbol{x}}\right)+\boldsymbol{N} .
$$

We assume no latency between $\tau_{e}$ and $\hat{\tau}_{e}$. By setting $\boldsymbol{M}_{v} \neq \boldsymbol{M}$ it is possible to shape inertia of both the attitude and position subsystems. The impedance control structure is shown in Fig. 5(a), and the system response along the inertial $x$-direction is shown in Fig. 5(b). The impedance controller has a simpler overall structure and is easier to tune than the admittance controller.

We note that with most flying systems (17) can not be obtained in the general case. The target dynamics (16) can only be obtained in the robot's control region. For a quadrotor, this means that impedance control can be achieved for the rotational dynamics and along the body $z$-axis only. Hence, for an impedance type behavior in the translational degrees of freedom we must use the attitude controller, as for position tracking control.

\section{Collision DETECTION AND REACTION}

Next, we investigate collisions of the flying robot with the environment. The external wrench estimate can be used for the four essential phases of collision Fault Detection, Identification and Isolation (FDI) [7], [8]. Collision detection provides binary information whether a collision with the environment has occurred. Collision classification provides information about the collision type. Through collision isolation we obtain the location of the collision. Lastly, we take appropriate collision reaction to mitigate danger.

a) Detection: A threshold on the estimated external force can be used as a collision detection signal. In this way, both slow contacts and impacts can be detected as collisions. However, sensitivity then depends on modeling errors and measurement noise. We can reduce their effect by applying a highpass filter $H(f)$ on the external force. Our collision detection signal $C D$ is then

$$
C D= \begin{cases}1 & \text { if } \exists i: H\left(\left|\hat{f}_{e, i}\right|\right)>f_{c, i} \\ 0 & \text { otherwise }\end{cases}
$$

In this way we can also distinguish contact from collisions.

b) Classification: Apart from the magnitude and shape information of the contact wrench, the collision force can in principle be used to also identify material properties of the colliding object. The force response of the continuous HuntCrossley contact model [17] is depicted in Fig. 6. Obviously the time and frequency response of the contact force depend on the material. Hence, in principle it is possible to classify the collision material from the contact force spectrum. It

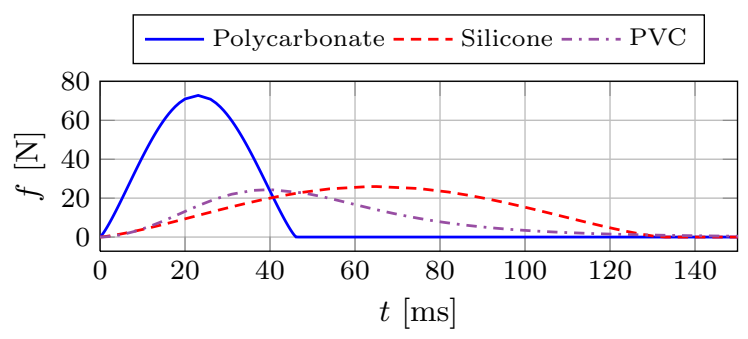

(a) Time domain response of the contact force on impact

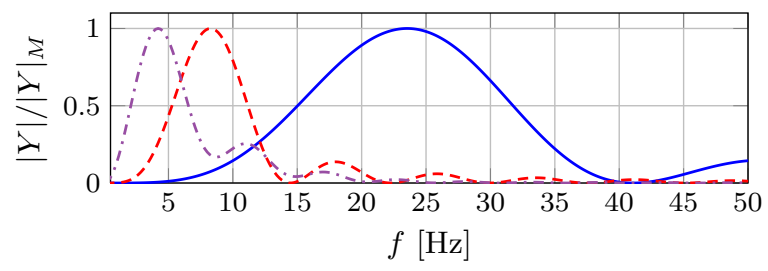

(b) Frequency characteristics of the contact force on impact

Fig. 6: Hunt-Crossley contact model for a point mass with $m=1$ $\mathrm{kg}$ at a collision velocity of $1 \mathrm{~m} / \mathrm{s}$. Different materials are shown [17], [18]. The impacting mass is $m=1 \mathrm{~kg}$. Stiffer materials have a higher impact frequency.

is possible to distinguish between materials only if the estimator frequency is larger than their frequency responses. As the treatment of collision classification is beyond the scope of this paper, we discuss the isolation of collision.

c) Isolation: contact position for tactile mapping: The contact location can be obtained from the estimated contact wrench during the contact phase. We assume that only a single contact point $\boldsymbol{r}_{c}$ exists, and that only a contact force is acting on the robot. The body-frame contact force $f_{e}^{b}$ then generates the torque $\boldsymbol{m}_{e}^{b}=\boldsymbol{r}_{c} \times \boldsymbol{f}_{e}^{b}$. This equation can not be solved in closed form for $\boldsymbol{r}_{c}$. However, the contact position lies on the ray

$$
\boldsymbol{r}_{c}=\boldsymbol{R}_{b i}\left(\frac{\hat{\boldsymbol{f}}_{e} \times \hat{\boldsymbol{m}}_{e}}{\left\|\hat{\boldsymbol{f}}_{e}\right\|^{2}}+\alpha \hat{\boldsymbol{f}}_{e}\right),
$$

where the free parameter $\alpha$ must be found. We find $\alpha$ by intersecting (19) with the robot's convex hull. For free-flight motion we can assume that the contact force will point into the robot, i.e. $\alpha<0$. This information can be used to estimate the collision surface that can be approximated with a plane. The collision plane in the inertial frame $\boldsymbol{n}_{p}^{T}\left(\boldsymbol{r}_{p}-\boldsymbol{o}\right)=$ 0 has the associated normal $\boldsymbol{n}_{p}=\hat{\boldsymbol{f}}_{e} /\left\|\hat{\boldsymbol{f}}_{e}\right\|$ and position $\boldsymbol{r}_{p}=\boldsymbol{r}+\boldsymbol{R}_{b i}^{T} \boldsymbol{r}_{c}$. This information could e.g. be stored in a contact map to prevent further collisions with static surfaces. The plane information may also be included in a more involved mapping solution, e.g. an octomap, and further used for (re-)planning.

To show the effectiveness of this method, a quadrotor is collided with a plane in simulation, for different collision speeds. The simulated contact with the plane occurs at a single point on the convex hull. For estimation purposes, the robot geometry is approximated using 8 rectangular surfaces. Fig. 7 shows the resulting plane estimation errors. It can be observed that the estimated surface normal is very accurate 


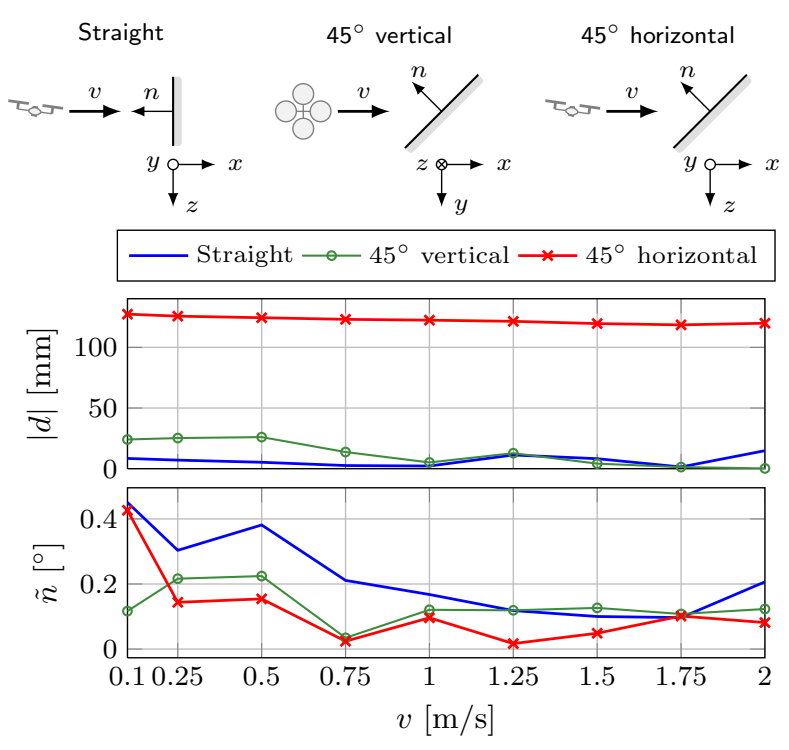

Fig. 7: Simulation of collision location determination for a quadrotor in different collision configurations. The upper plot shows the absolute distance of the estimated plane position to the real plane $d=\boldsymbol{n}^{T}\left(\boldsymbol{r}_{p}-\boldsymbol{o}\right)$. The lower plot shows the angular error of the estimated normal $\tilde{n}=\operatorname{acos} \boldsymbol{n}^{T} \boldsymbol{n}_{p}$. The mean of multiple collisions is shown for each velocity. The standard deviation in both cases too small to be visible.

for the entire velocity range and all impact configurations. The maximum standard deviation of the angle error $\tilde{n}$ is less than $10^{-3} \mathrm{deg}$ in magnitude. The position determination accuracy depends on the impact angle and the used geometric model. Even for the very approximate geometric model, for straight collisions the accuracy is better than $1 \mathrm{~cm}$. The maximum standard deviation of all cases is $2 \mathrm{~mm}$. The accuracy is consistent across collision velocities. The error of the $45^{\circ}$ horizontal case is due to geometric approximation. The approximated robot's bottom surface is in fact already 5 $\mathrm{cm}$ below the actual contact point, which obviously causes a systematic error in the position estimation.

d) Collision reaction: Now, we investigate several basic collision reflex reaction strategies (i.e. no planning). Figure 8 depicts the inertial $x$-axis trajectory of a quadrotor colliding with a stiff surface (polycarbonate). The surface is located at $x=1 \mathrm{~m}$, and the collision occurs at a velocity of $1 \mathrm{~m} / \mathrm{s}$. The quadrotor arm is $27 \mathrm{~cm}$ long.

No reaction. When no control reaction is done upon collision, the quadrotor becomes unstable and could crash.

Trajectory Stop. Upon collision, the trajectory is stopped at the current reference position. This halts the robot approximately at the obstacle position.

Equilibrium Bounce. Upon detecting a collision, we set the equilibrium position to $\boldsymbol{r}_{d}=\boldsymbol{r}_{k}+\delta_{c} \hat{\boldsymbol{f}}_{e}^{0}$ where $\boldsymbol{r}_{k}$ is the robot position at the time of collision, and $\delta_{c}>0$ is the bounce distance. This will drive the path backwards along the direction of the collision normal. The robot will then stabilize at a safe distance from the obstacle.

Collision force amplification. The controller is switched to impedance or admittance mode immediately upon a collision. We perform only damping, i.e. $\boldsymbol{K}_{v}=\mathbf{0}$. The

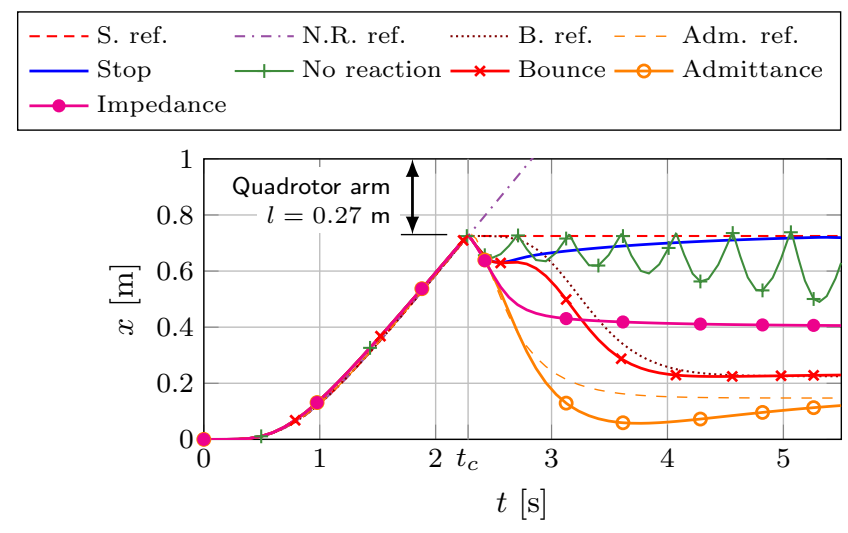

Fig. 8: Simulated robot trajectories for different collision reaction strategies. Without reaction, the robot might become unstable. A stop reaction halts the robot at the collision position. A bounce reaction with $\delta_{c}=0.5 \mathrm{~m}$ sets the equilibrium position in the opposite direction of the collision normal. The interaction controllers $\left(K_{r, x}=1\right)$ show very fast reaction. The system mass is $m=0.55$ $\mathrm{kg}$, and the virtual mass is set to $m_{v}=0.25 \mathrm{~kg}$. Damping during admittance control is $d_{v, x}=3 \mathrm{Ns} / \mathrm{m}$, and during impedance control it is $d_{v, x}=0.25 \mathrm{Ns} / \mathrm{m}$.

input to the control system is the amplified collision force $\boldsymbol{\tau}_{v}=\boldsymbol{K}_{r} \hat{\boldsymbol{\tau}}_{e}$. For admittance control we then have

$$
\boldsymbol{M}_{v} \ddot{\boldsymbol{x}}_{v}+\boldsymbol{D}_{v} \dot{\boldsymbol{x}}_{v}=\boldsymbol{K}_{r} \hat{\boldsymbol{\tau}}_{e},
$$

and for impedance control we have

$$
\boldsymbol{J}^{T} \boldsymbol{\tau}=\left(\boldsymbol{M} \boldsymbol{M}_{v}^{-1}-\boldsymbol{I}\right) \boldsymbol{K}_{r} \hat{\boldsymbol{\tau}}_{e}-\boldsymbol{M} \boldsymbol{M}_{v}^{-1} \boldsymbol{D}_{v} \tilde{\boldsymbol{\nu}}+\boldsymbol{N} .
$$

This essentialy produces an energy disspation reflex. The reaction is in both cases significantly faster than trajectorybased approaches.

e) Application: take-off and landing detection: Takeoff and landing events typically occur on horizontal surfaces. When landed, the external force is of course the robot weight $W=m g$, see Fig. 3. The external force along the inertial $z$-direction can therefore be monitored to detect take-off and landing, e.g. in the form of a threshold. This information can also be used to check the consistency of a landing. If the wrench acting on the robot becomes statically inconsistent, recovery behavior can be employed.

\section{EXPERIMENTAL RESULTS}

a) Setup: Experiments are performed using an AscTec Hummingbird quadrotor with our attitude controller running onboard. The position controller, external wrench estimation and related functions are running on a remote PC in Simulink. The attitude commands are sent to the quadrotor using XBee communication. Position and attitude are obtained from an ART motion capture system. The attitude control loop runs at $1 \mathrm{kHz}$, and the position control loop runs at $60 \mathrm{~Hz}$. Table II lists the used system parameters. The used XBee communication is insufficient for two-way communication. We therefore unfortunately can not receive IMU data from the onboard sensors at the moment. For estimation purposes, these are obtained from the motion capture data. This results in significantly more noisey acceleration 
TABLE II: System parameters used in the experiments.

\begin{tabular}{|l|l|l|}
\hline Parameter & Value & Unit \\
\hline \hline$m$ & 0.55 & $\mathrm{~kg}$ \\
$\boldsymbol{K}_{p}$ & $\operatorname{diag}\{2.25,2.25,5.06\}$ & $\mathrm{N} / \mathrm{m}$ \\
$\boldsymbol{K}_{d}$ & $\operatorname{diag}\{3,3,4.5\}$ & $\mathrm{N} \mathrm{s} \mathrm{m}^{-1}$ \\
\hline $\boldsymbol{I}$ & $\operatorname{diag}\{3,3,4\}$ & $10^{-3} \mathrm{~kg} \mathrm{~m}^{2}$ \\
$c$ & 256 & - \\
$\boldsymbol{K}_{\omega}$ & $\operatorname{diag}\{32,32,32\}$ & $\mathrm{N} \mathrm{m} \mathrm{sad}^{-1}$ \\
\hline
\end{tabular}

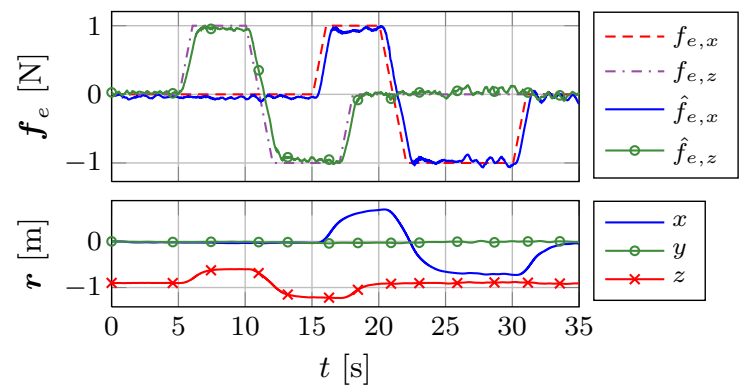

Fig. 9: External force estimation in flight with $K_{I}^{f}=3$. The force is applied virtually, so that ground truth information can be provided.

and angular velocity signals. Necessary filtering then results in slower estimator dynamics and reflex reaction.

b) External force estimation: An external force is applied to the quadrotor while being in hovering mode. We simulate a virtual external force acting on the robot, so that ground truth information is available. Fig. 9 shows the external force estimation in the inertial $x$ - and $z$-directions during hover. Clearly, the estimated force is a lowpassfiltered signal of the applied force, as expected.

c) Impedance control: Due to technical constraints, we were only able to implement an impedance controller for the position subsystem, as depicted in Fig. 10(a). Of course, in the future we plan to redesign the control structure to obtain the controller as in Section IV-B. The impedance controller for the position subsystem has the form

$$
\boldsymbol{f}_{i}=\left(m m_{v}^{-1}-1\right) \hat{\boldsymbol{f}}_{e}-m m_{v}^{-1}(\hat{d} \dot{\tilde{\boldsymbol{r}}}+\hat{k} \tilde{\boldsymbol{r}})-m g \boldsymbol{e}_{3},
$$

and uses the attitude controller as in Fig. 1. Effectively, the rotational inertia is not scaled, i.e. $\boldsymbol{I}_{v}=\boldsymbol{I}$. During the experiment, the controller was in damping mode $\left(\boldsymbol{K}_{v}=\mathbf{0}\right)$, and a human has applied a force to the robot. The position response and estimated force during interaction are shown in Fig. 10(b).

d) Collision reaction and location: A polystyrene block was placed on the robot's flight path and used as an obstacle. The position response of different collision reaction schemes along the inertial $x$-direction is depicted in Fig. 11. The obstacle is static between the experiments. We used the highpass filtered external force as a collision detection signal. It can be seen that even for a highly filtered external force, the presented schemes are effective. Without a reaction scheme, the robot became unstable and crashed. The trajectory-based schemes (stop, bounce) successfully stabilized the quadrotor after a collision. Switching to impedance damping mode provides the fastest and smoothest response to the collision. The estimated obstacle locations is shown in Table III. It

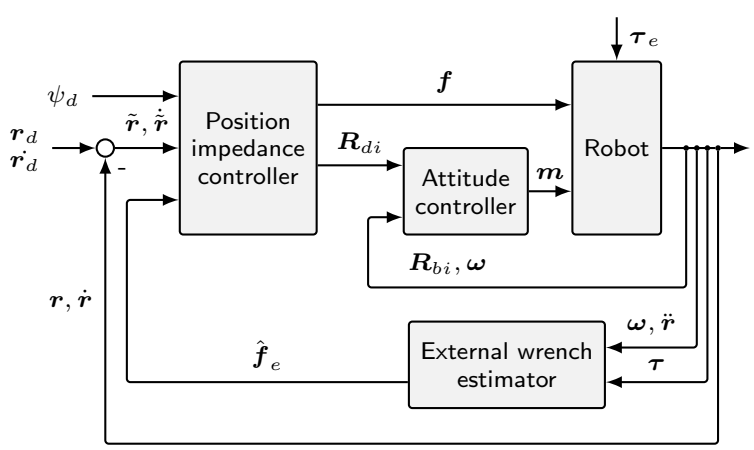

(a) Control structure of the implemented impedance controller.

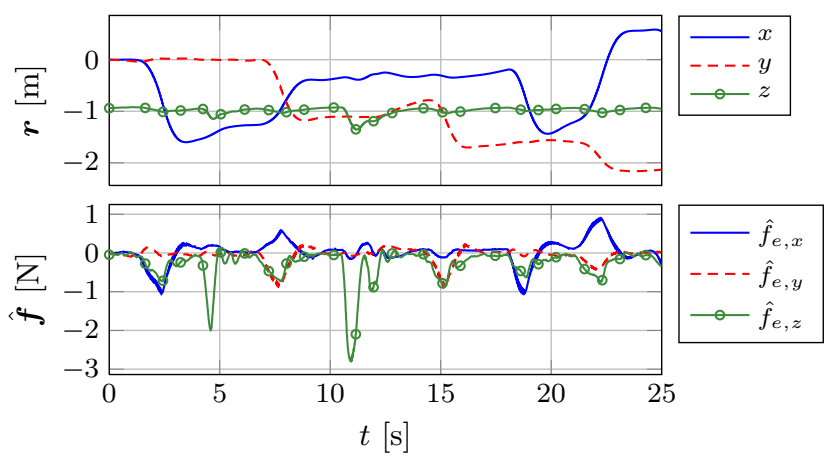

(b) Trajectory an estimated external force in the impedance controller experiment.

Fig. 10: Impedance controller experiment with $m_{v}=0.25 \mathrm{~kg}$, $\boldsymbol{D}_{v}=\operatorname{diag}\{1,1,1\} \mathrm{N} / \mathrm{s}, \boldsymbol{K}_{v}=\operatorname{diag}\{0,0,2\} \mathrm{N} / \mathrm{m}$. The quadrotor can be freely moved around along the inertial $x$ and $y$ axes.

TABLE III: Estimated obstacle location and associated surface normal in the collision reaction experiment. The position is expressed in meters, in the inertial frame.

\begin{tabular}{|l|lll|lll|}
\hline Reaction & $x$ & $y$ & $z$ & $n_{x}$ & $n_{y}$ & $n_{p}$ \\
\hline \hline No reaction & 1.129 & -0.339 & -0.841 & -0.964 & 0.051 & -0.262 \\
Stop & 1.140 & -0.335 & -0.864 & -0.983 & -0.006 & -0.180 \\
Bounce & 1.139 & -0.242 & -0.851 & -0.982 & -0.072 & -0.175 \\
Impedance & 1.145 & -0.304 & -0.834 & -0.989 & 0.034 & -0.145 \\
\hline
\end{tabular}

can be seen that the obstacle plane estimation is precise and consistent across experiments.

e) Takeoff and landing: Lastly, Figure 12 shows a complete flight with multiple bounce collision reactions. Individual events can be clearly seen from the external forces.

\section{CONCLUSION}

Contact wrench information together with interaction control schemes is very valuable during physical interaction of a robot and its environment. In this paper we have solved the problem of obtaining external wrench information in flying robots without the need of additional sensors. Furthermore, we developed a set of interaction control schemes based on well established admittance and impedance concepts. This has enabled several applications under a unified framework. In particular, we have investigated interaction controllers and collisions with the environment. We show that sensitive interaction control together with fast collision reflexes significantly enrich the robot's behavior. Also, the complete wrench 

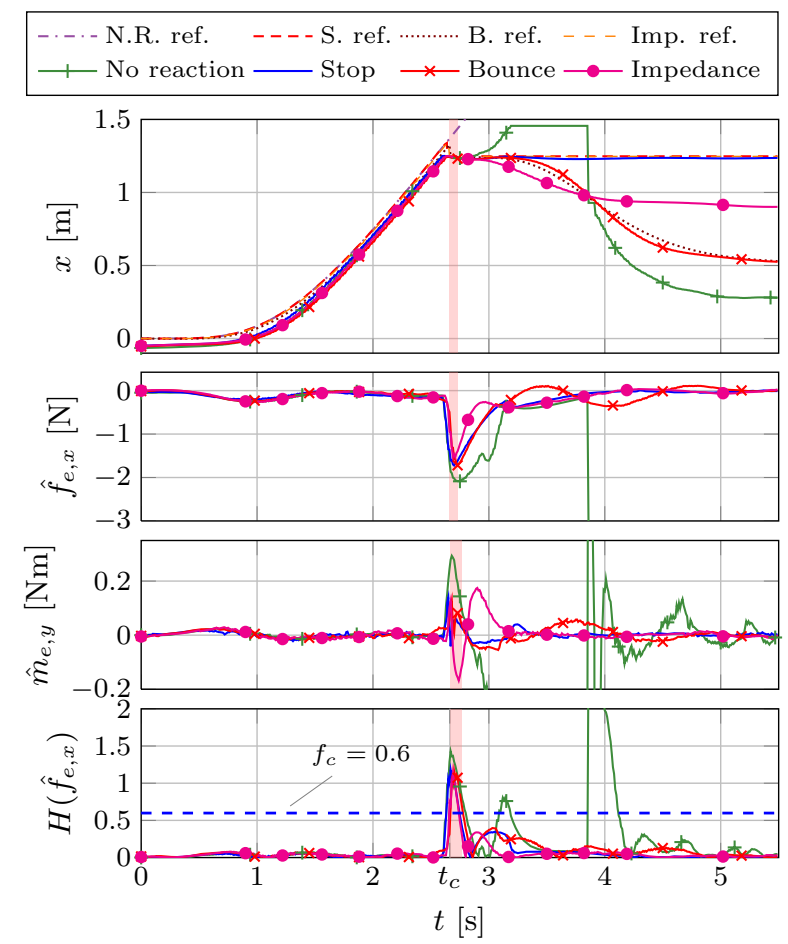

Fig. 11: Collision reaction experiment with a polystyrene block. From top to bottom: position, estimated external force, estimated external pitch torque, collision detection signal. The force estimate is filtered with $K_{I}^{f}=3$, and torque with $K_{I}^{m}=12$. The collision detection threshold is set to $f_{c}=0.6$. Collisions are detected at approx. $t_{c}$. The red shaded area depicts the contact phase. The quadrotor crashed when the collision was not accounted for. All reaction schemes stabilize the robot upon collision. Impedance mode with collision force augmentation provides the fastest response. Here we used $m_{v}=0.25 \mathrm{~kg}, d_{v}=0.5 \mathrm{Ns} / \mathrm{m}$, and $K_{r, x}=1$.

information allows us to additionally map the envionment upon collisions. Together, our contributions are a significant step towards enabling flying robots to operate safely and robustly in cluttered environments.

Important to notice on a technical level, our novel hybrid method for estimating the external wrench requires only proprioceptive measurements from an onboard IMU, a dynamical model and the control input. In contrast to previous methods, it is independent of the used controller and the desired trajectory. Collision detection, isolation, classification and reactions make use of the full external wrench information. No specific mechanical design is required for collision robustness.

Next steps are a thorough stability analysis of the interaction controllers that use the external wrench estimate in closed loop. We also plan to use the contact position determination in a complex autonomous exploration scenario.

\section{REFERENCES}

[1] S. Bellens, J. De Schutter, and H. Bruyninckx, "A hybrid pose / wrench control framework for quadrotor helicopters," in ICRA 2012, 2012, pp. 2269-2274.

[2] H.-N. Nguyen and D. Lee, "Hybrid force/motion control and internal dynamics of quadrotors for tool operation," in IROS 2013, Tokyo, Japan, Nov 2013, pp. 3458-3464.

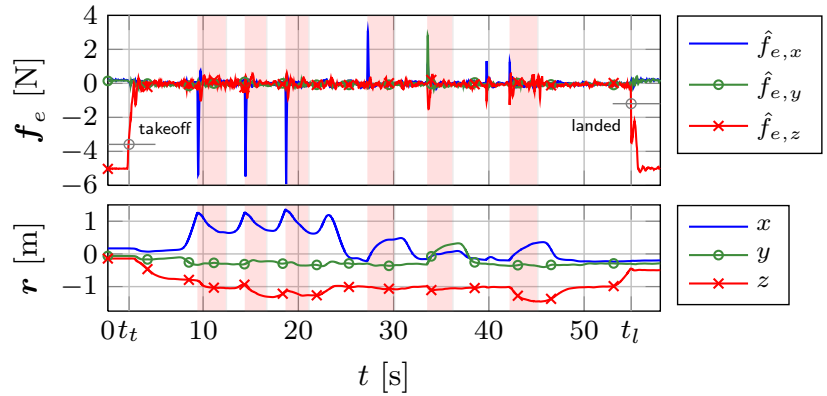

Fig. 12: Flight with multiple collisions. Equilibrium bounce reactions are indicated by shaded areas. Normal flight is resumed after stabilization. After $t=25 \mathrm{~s}$, the quadrotor hovers and is hit by a human. Landed phases are clearly visible from $\hat{f}_{e, z}$. Takeoff is detected at $t=t_{t}$, and landing at $t=t_{l}$. External force estimation in flight was performed with $K_{I}^{f}=50$.

[3] S. Jung, "A Position-Based Force Control Approach to a Quad-rotor System," in Ubiquitous Robots and Ambient Intelligence (URAI), 2012 9th International Conference on, 2012, pp. 373-377.

[4] M. Fumagalli, R. Naldi, A. Macchelli, R. Carloni, S. Stramigioli, and L. Marconi, "Modeling and control of a flying robot for contact inspection," in IROS 2012, 2012, pp. 3532-3537.

[5] M. Fumagalli and R. Carloni, "A modified impedance control for physical interaction of UAVs," in IROS 2013, 2013, pp. 1979-1984.

[6] K. Schmid, T. Tomić, F. Ruess, H. Hirschmüller, and M. Suppa, "Stereo vision based indoor/outdoor navigation for flying robots," in IROS 2013, 2013, pp. 3955-3962.

[7] S. Haddadin, A. Albu-Schaffer, A. De Luca, and G. Hirzinger, "Collision detection and reaction: A contribution to safe physical humanrobot interaction," in IROS 2008, Sept 2008, pp. 3356-3363.

[8] S. Haddadin, Towards Safe Robots - Approaching Asimov's 1st Law, ser. Springer Tracts in Advanced Robotics. Springer Verlag, 2014, vol. 90.

[9] A. Albers, S. Trautmann, T. Howard, T. A. Nguyen, M. Frietsch, and C. Sauter, "Semi-autonomous flying robot for physical interaction with environment," in Robotics Automation and Mechatronics (RAM), 2010 IEEE Conference on, 2010, pp. 441-446.

[10] F. Forte, R. Naldi, A. Macchelli, and L. Marconi, "Impedance control of an aerial manipulator," in American Control Conference (ACC), 2012, 2012, pp. 3839-3844.

[11] R. Naldi, A. Torre, and L. Marconi, "Robust blind navigation for a miniature ducted-fan aerial robot," in American Control Conference (ACC), 2013, 2013, pp. 988-993.

[12] A. Briod, P. Kornatowski, A. Klaptocz, A. Garnier, M. Pagnamenta, J.-C. Zufferey, and D. Floreano, "Contact-based navigation for an autonomous flying robot," in IROS 2013, 2013, pp. 3987-3992.

[13] T. Lee, M. Leoky, and N. McClamroch, "Geometric tracking control of a quadrotor UAV on SE(3)," in Decision and Control (CDC), 2010 49th IEEE Conference on, Dec 2010, pp. 5420-5425.

[14] A. De Luca, A. Albu-Schaffer, S. Haddadin, and G. Hirzinger, "Collision Detection and Safe Reaction with the DLR-III Lightweight Manipulator Arm," in IROS 2006, 2006, pp. 1623-1630.

[15] D. Honegger, L. Meier, P. Tanskanen, and M. Pollefeys, "An open source and open hardware embedded metric optical flow cmos camera for indoor and outdoor applications," in ICRA 2013, 2013, pp. 17361741.

[16] N. Hogan, "Impedance control - An approach to manipulation. I Theory. II - Implementation. III - Applications," ASME Transactions Journal of Dynamic Systems and Measurement Control B, vol. 107, pp. 1-24, Mar. 1985.

[17] N. Diolaiti, C. Melchiorri, and S. Stramigioli, "Contact impedance estimation for robotic systems," Robotics, IEEE Transactions on, vol. 21, no. 5, pp. 925-935, 2005.

[18] A. Haddadi and K. Hashtrudi-Zaad, "Real-Time Identification of HuntCrossley Dynamic Models of Contact Environments," Robotics, IEEE Transactions on, vol. 28, no. 3, pp. 555-566, June 2012. 